Neutrino Physics and Neutrino Astrophysics

\author{
Gail McLaughlin
}

North Carolina State 


\section{Properties of Neutrinos}

\begin{tabular}{|l|c|}
\hline \multirow{2}{*}{ Mass? } & $\begin{array}{l}m_{\nu_{e}}<2 \mathrm{eV} \text { from tritium } \beta \text { decay } \\
m_{\nu_{\mu}}<170 \mathrm{keV} \text { from } \pi \text { decay } \\
m_{\nu_{\tau}}<18 \mathrm{MeV} \text { from } \tau \text { decay }\end{array}$ \\
\hline Spin & $s=1 / 2$ \\
\hline Type? & $\begin{array}{c}\text { Dirac } \nu \neq \bar{\nu} \\
\text { Majorana } \nu=\bar{\nu}\end{array}$ \\
\hline Charge & $Q=0$ \\
\hline Interactions & weak (and gravitational) only \\
\hline Flavors & $\begin{array}{l}3 \text { active flavors (from } Z \text { width) } \\
\text { sterile flavors? }\end{array}$ \\
\hline
\end{tabular}




\section{Direct Measurements: Tritium $\beta$ Decay}

tritium beta decay

$$
\begin{gathered}
{ }^{3} \mathrm{H} \rightarrow{ }^{3} \mathrm{He}+e^{-}+\bar{\nu}_{e} \\
E_{0}=18.6 \mathrm{keV}, \quad T_{1 / 2}=12.3 a^{\circ / 2}
\end{gathered}
$$

Neutrino mass modifies electron spectrum near end point

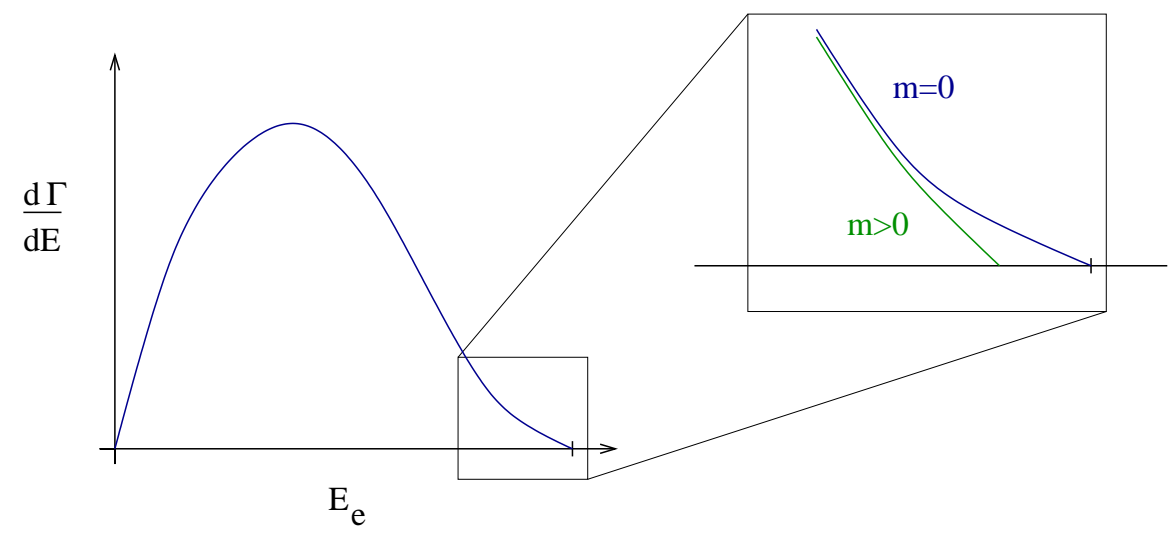

Mainz neutrino mass experiment (1998-2001)

$$
m_{\nu_{e}}<2.2 \mathrm{eV} \quad(95 \% \mathrm{CL})
$$




\section{Double Beta Decay}

$2 \nu 2 \beta$ vs $0 \nu 2 \beta$ decay
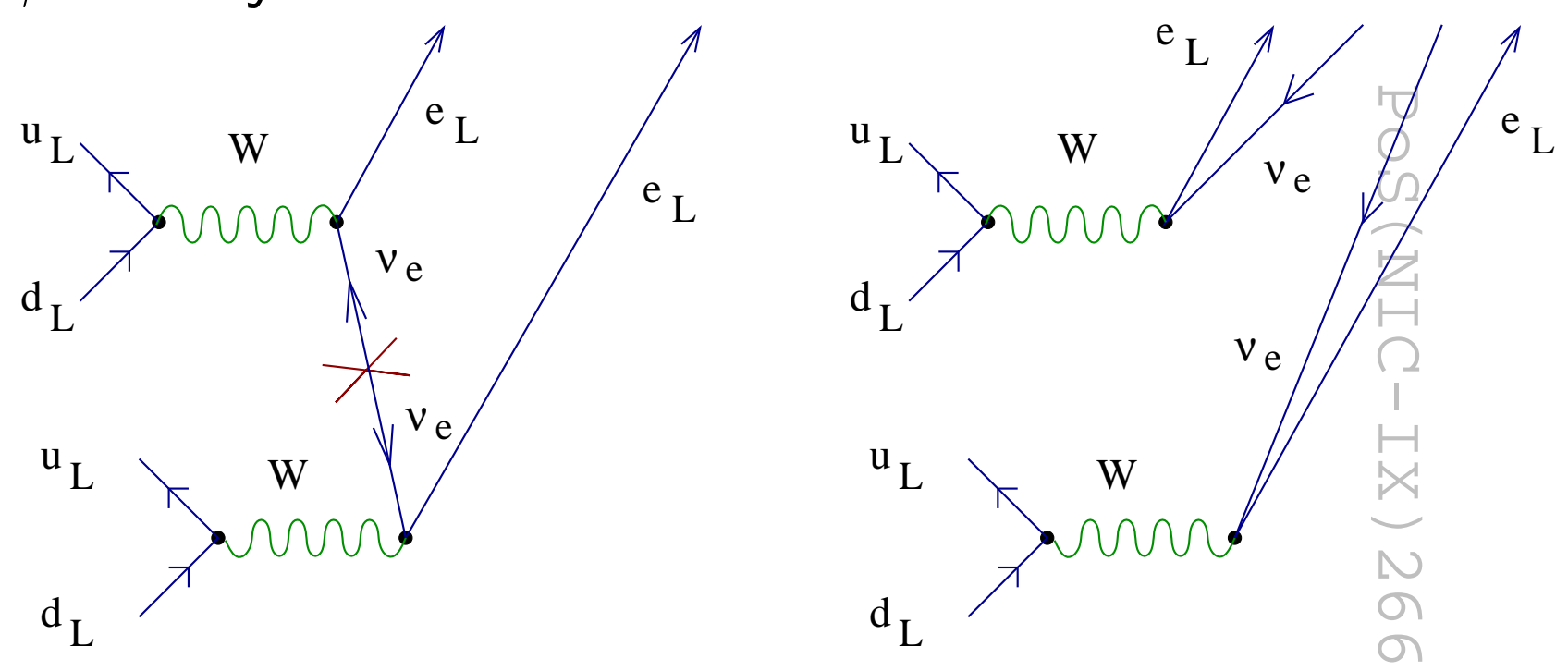

$\left(T_{1 / 2}^{0 \nu}\right)^{-1} \sim($ phase space $) \times(\text { nuclear m.e. })^{2} \times\left\langle m_{\nu}^{M}\right\rangle^{2}$

$$
\left\langle m_{\nu}^{M}\right\rangle=\left|\Sigma_{k} U_{e k}^{2} m_{k}^{M}\right|
$$

where $U_{e k}$ is the MNS matrix 
need nucleus which is $\beta$ stable, but $2 \beta$ unstable
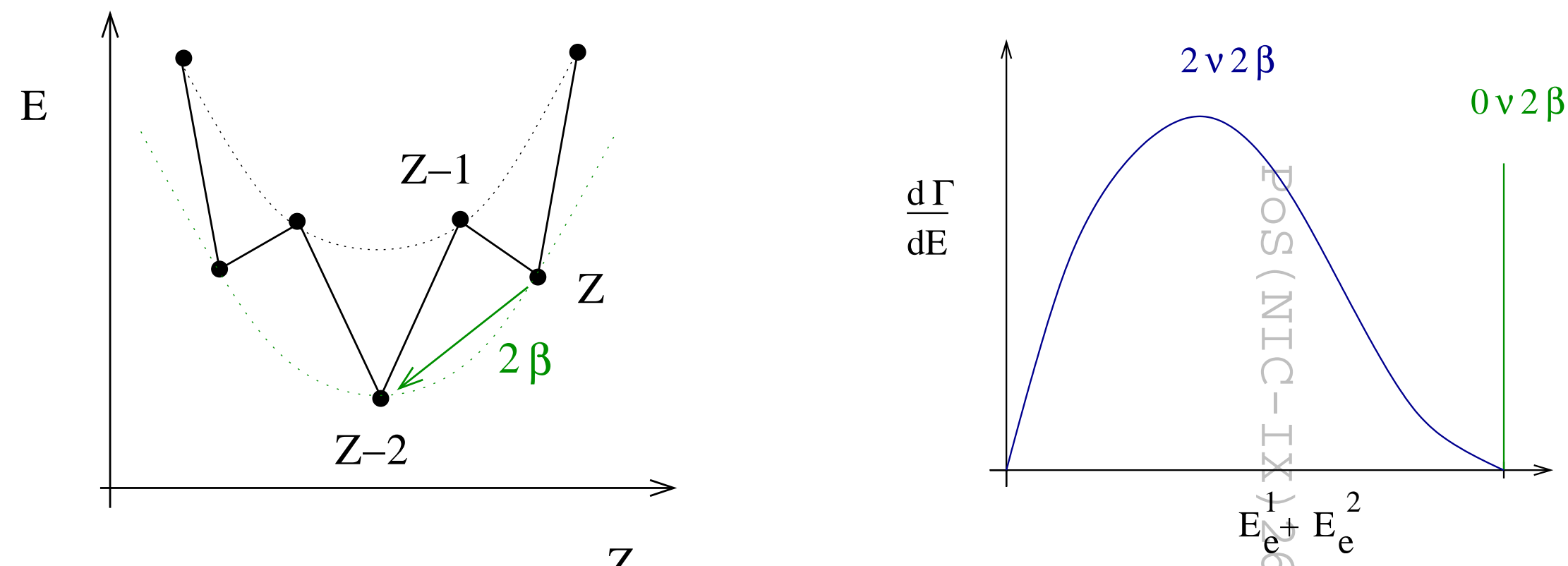

$\mathrm{Z}$

look for events with $E_{e}^{1} f E_{e}^{2}=Q$

Heidelberg-Moscow (1999-2000)

${ }^{76} \mathrm{Ge} \quad T_{1 / 2}^{0 \nu}>1.9 \cdot 10^{25} \mathrm{yr} \quad\left\langle m_{\nu}\right\rangle<0.35 \mathrm{eV}$ 


\section{Neutrino Dark Matter}

Early universe: photons in thermal equilibrium

$$
e^{+}+e^{-} \leftrightarrow \gamma+\gamma, \quad \gamma+e^{-} \leftrightarrow \gamma+e^{-}, \ldots
$$

Photons decouple at $T_{\gamma}^{\text {recom }}=4000 \mathrm{~K}$, get red-shifted to

$$
T_{\gamma}^{\text {now }}=2.7 \mathrm{~K}
$$

Energy density given by Stefan-Boltzmann law

$$
\rho_{\gamma}=4 \frac{\pi^{2}}{60} T_{\gamma}^{4}=4 \cdot 10^{-34} \mathrm{~g} \mathrm{~cm}^{-3}
$$

Early universe: neutrinos equilibrate via

$$
e^{+}+e^{-} \leftrightarrow \nu_{e}+\bar{\nu}_{e}, \quad \nu_{e}+e^{-} \leftrightarrow \nu_{e}+e^{-}, \ldots
$$

Neutrinos decouple at $T_{\nu}^{d e c}=10^{10} \mathrm{~K}$. 
Photons get reheated by $e^{+}+e^{-} \rightarrow \gamma \gamma$. Today

$$
T_{\nu}=T_{\gamma}\left(\frac{4}{11}\right)^{1 / 3}=1.94 \mathrm{~K}
$$

Energy density of massless neutrinos

$$
\rho_{\nu}=\frac{7}{2} N_{\nu} \frac{\pi^{2}}{60} T_{\nu}^{4}=0.7 \rho_{\gamma} \quad\left(N_{\nu}=3\right)^{2}
$$

Number density today $n_{\nu} \sim 330 \mathrm{~cm}^{-3}$.

Massive neutrinos contribute to the energy density of the universe. If neutrinos relativistic at decoupling then the number density is not affected by $m$. Energy density

$$
\rho_{\nu}=m n_{\nu}
$$


Simplest constraint:

\section{universe is flat $\rightarrow$ restricts the neutrino mass}

Energy budget of the universe

$$
\begin{gathered}
\Omega_{D M} \approx 0.23 \pm 0.04 \\
\Omega_{\Lambda}=0.73 \pm 0.04 \\
\Omega_{B}=0.044 \pm 0.004 \\
\Omega_{\nu}=0.02\left(\sum \frac{m_{\nu}}{1 \mathrm{eV}}\right)\left(\frac{72 \mathrm{~km} \mathrm{sec}^{-1} \mathrm{Mpc}^{-1}}{H_{0}}\right)^{2} \\
m_{\nu_{e}}+m_{\nu_{\mu}}+m_{\nu_{\tau}}<25 \mathrm{eV}
\end{gathered}
$$




\section{Power spectrum of background radiation (WMAP)}

\section{provides much stronger constraints}

$$
m_{\nu_{e}}+m_{\nu_{\mu}}+m_{\nu_{\tau}}<1 \mathrm{eV}
$$

How? One way is that $m_{\nu}$ changes the expansion rate of the universe

At the surface of last scattering, this leads to a change in the sound horizon

This changes the position of peaks and troughs in the anisotropy spectrum which is measured by WMAP 


\section{Another avenue: Power spectrum + large scale structure}

Neutrino mass also changes large scale structure

On small scales $\left(\lambda_{f s}<1 \mathrm{Mpc}\left(\frac{1 \mathrm{eV}}{\mathrm{m}_{\nu}}\right)(1+z)^{1 / 2}\right)$ it damps the growth of large scale structure since it can freely stream in and out of small density perturbations (larger thermal speed of the neutrino as opposed to the dark matter)

On large scales it acts like dark matter

Combination analysis of WMAP and large scale structure could put limits on the sum of the active neutrino masses of tenths of an $\mathrm{eV}$. 


\section{Where are we with 'direct' measures of neutrino mass?}

1. $m_{\nu_{e}}<2.2 \mathrm{eV}$ from ${ }^{3} \mathrm{H}$ decay

2. No detection yet from double beta decay

3. From the Cosmic Microwave Background $\sum m_{\nu}<1 \mathrm{eV}$ 


\section{High Energy Astrophysical Neutrinos}

Atmospheric neutrino flux dies off at $10^{14}-10^{15} \mathrm{eV}$

What is beyond?

1. Look for sources in the distant high energy universe

$\rightarrow$ photons absorbed

$\rightarrow$ cosmic rays deflected from magnetic fields

$\rightarrow$ but the neutrinos survive

2. Exotic Sources $\rightarrow$ dark matter annihilation and decay

3. GZK neutrinos $\rightarrow$ Neutrinos from Cosmic Rays

How to detect? Ice Cube, ANITA, etc... 


\section{GZK neutrinos}

Origin and Nature of the Cosmic Rays

Particles are accelerated by extragalactic sources

Proton hits microwave background photon $p+\gamma \rightarrow n+\pi^{-}$, $\pi^{-} \rightarrow \mu^{-}+\bar{\nu}_{\mu}, \mu^{-} \rightarrow e^{-} \bar{\nu}_{e} \nu_{\mu}$

This is the "GZK effect" and it sets in about $4 \times 10^{19} \mathrm{eV}$.

Look for the $10^{18} \mathrm{eV}$ neutrinos from this scattering in $\mathrm{km}^{3}$ detectors.

Event rates for neutrinos about $10^{15} \mathrm{eV}$ are predicted to be at 0.1 to 1 event per $\mathrm{km}^{3}$ per year. 


\section{Supernova Neutrinos}

Most neutrinos emitted during the first $\sim 10 \mathrm{sec}$

Galactic supernovae estimated to occur $\sim 1$ every 30 years Supernova neutrinos detected from SN1987a:

$\sim 20$ events observed in Kamiokande and IMB.

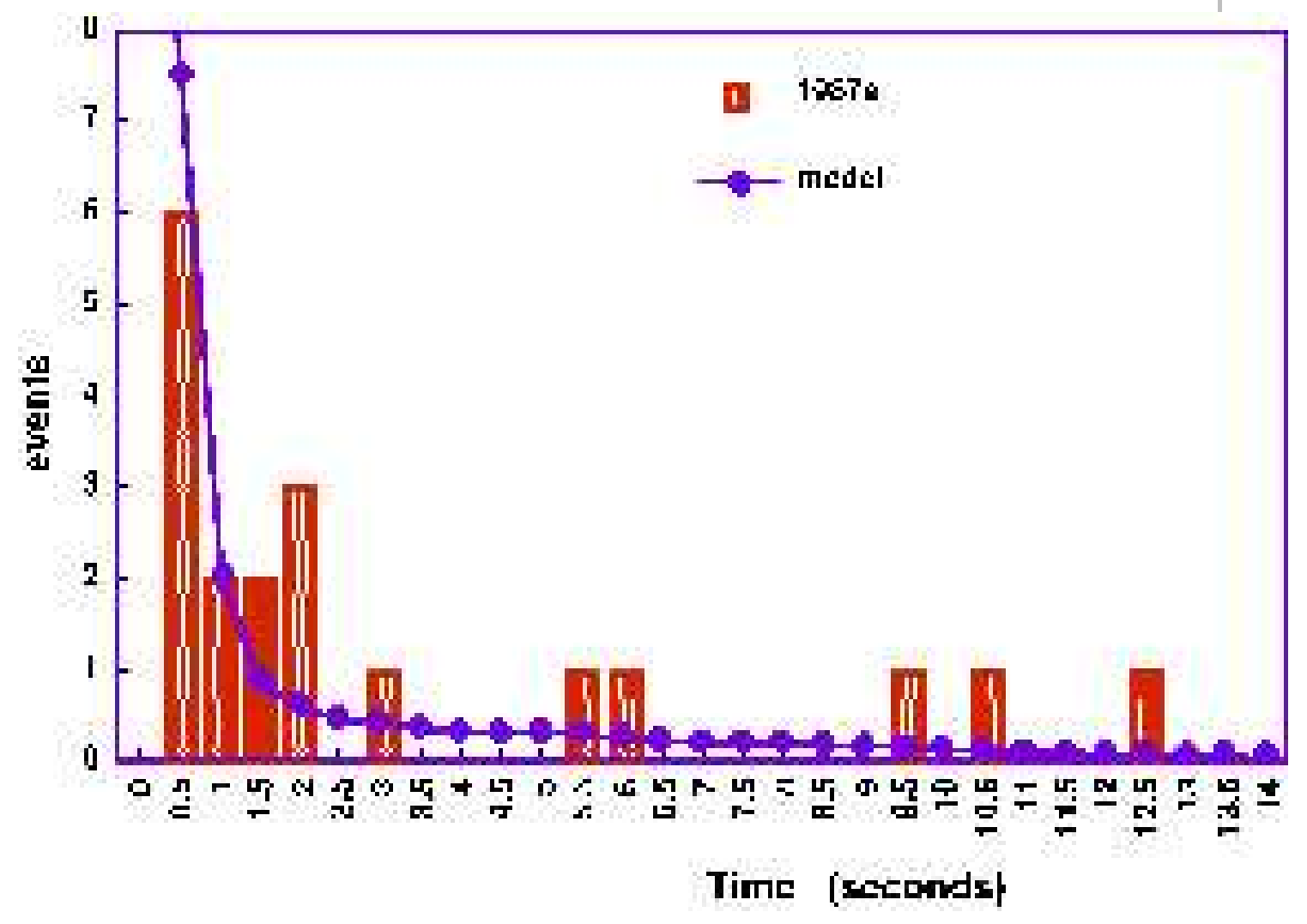


Current detectors will record 1000's of events

SuperK: $\quad \sim 8000 \bar{\nu}_{e}+p \rightarrow e^{+}+n$ évents

SNO: $\quad \sim 500 \quad \nu+d \rightarrow \nu+p+n$

SNO: $\quad \sim 80 \quad \nu_{e}+d \rightarrow e^{-}+p+{ }_{\circ} n$

KamLAND: $\sim 300 \quad \bar{\nu}_{e}+p \rightarrow n+e^{+}$

KamLAND: $\sim 100 \mathrm{~s} \quad \nu+p \rightarrow \nu+p$ 


\section{Supernova Neutrinos}

All types of neutrinos emanate from the proto-neutron star core. They travel through the outer layers of the SN, then to earth.

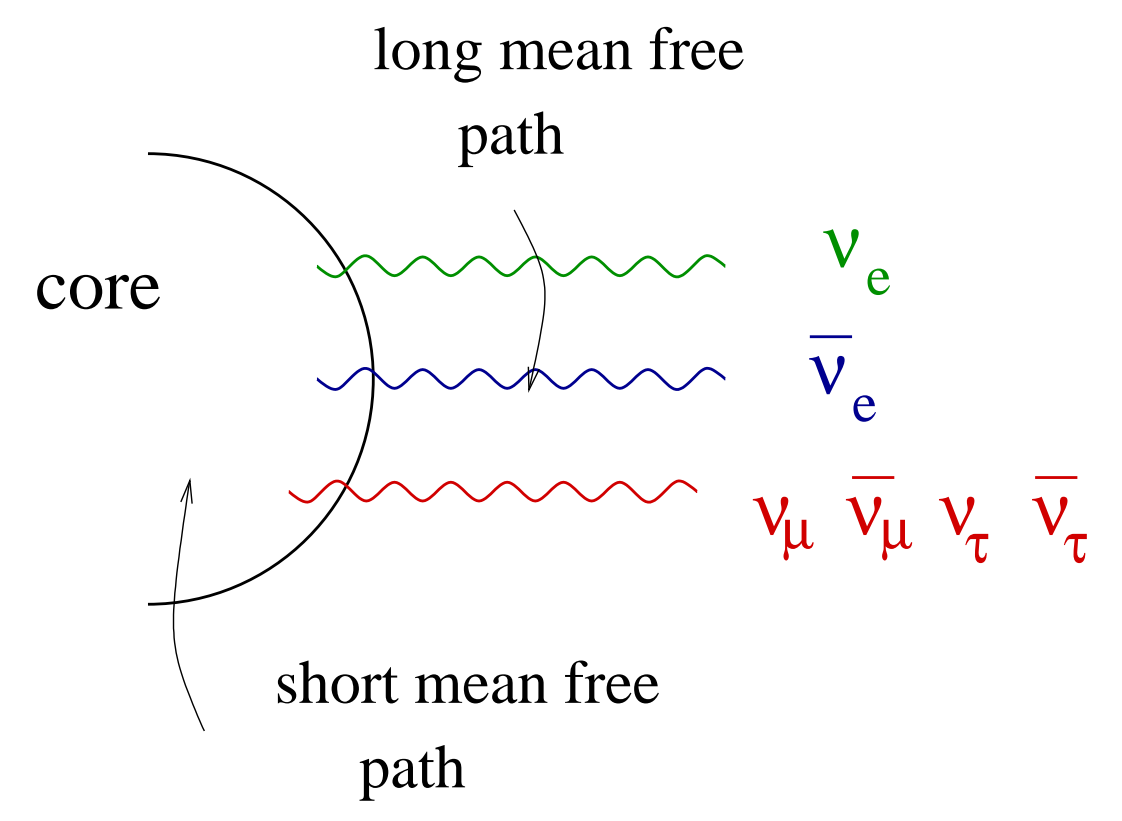




\section{Measuring the Supernova Neutrino Signal}

Why? Neutrinos are our window deep into the core of the Supernova

Different neutrino transport calculations predict different spectra

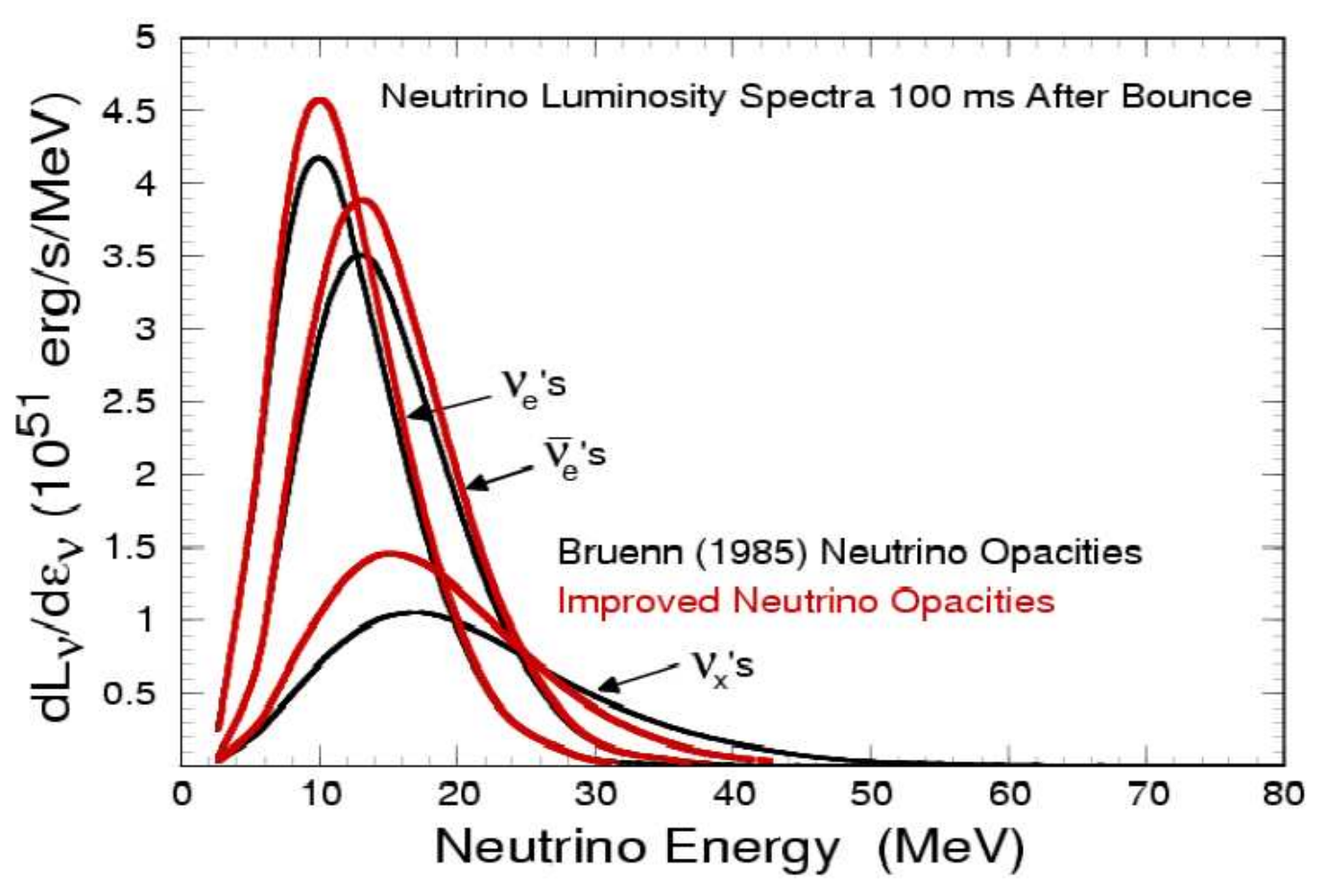

Roughly the range is

- $\left\langle E_{\nu_{\mu}}\right\rangle=\left\langle E_{\bar{\nu}_{\mu}}\right\rangle=\left\langle E_{\nu_{\tau}}\right\rangle$ $=\left\langle E_{\bar{\nu}_{\tau}}\right\rangle \stackrel{Ð}{\circledast} 20-30 \mathrm{MeV}$

- $\left\langle E_{\bar{\nu}_{e}}\right\rangle=13-19 \mathrm{MeV}$

- $\left\langle E_{\nu_{e}}\right\rangle=8-13 \mathrm{MeV}$

The shape is different, too. 


\section{Do all core-collapse events produce these neutrinos?}

- Actually, not all core-collapse are predicted to produce proto-neutron stars

- Some are predicted to produce black holes

- And a very small number are predicted to produce accretion disks around black holes...

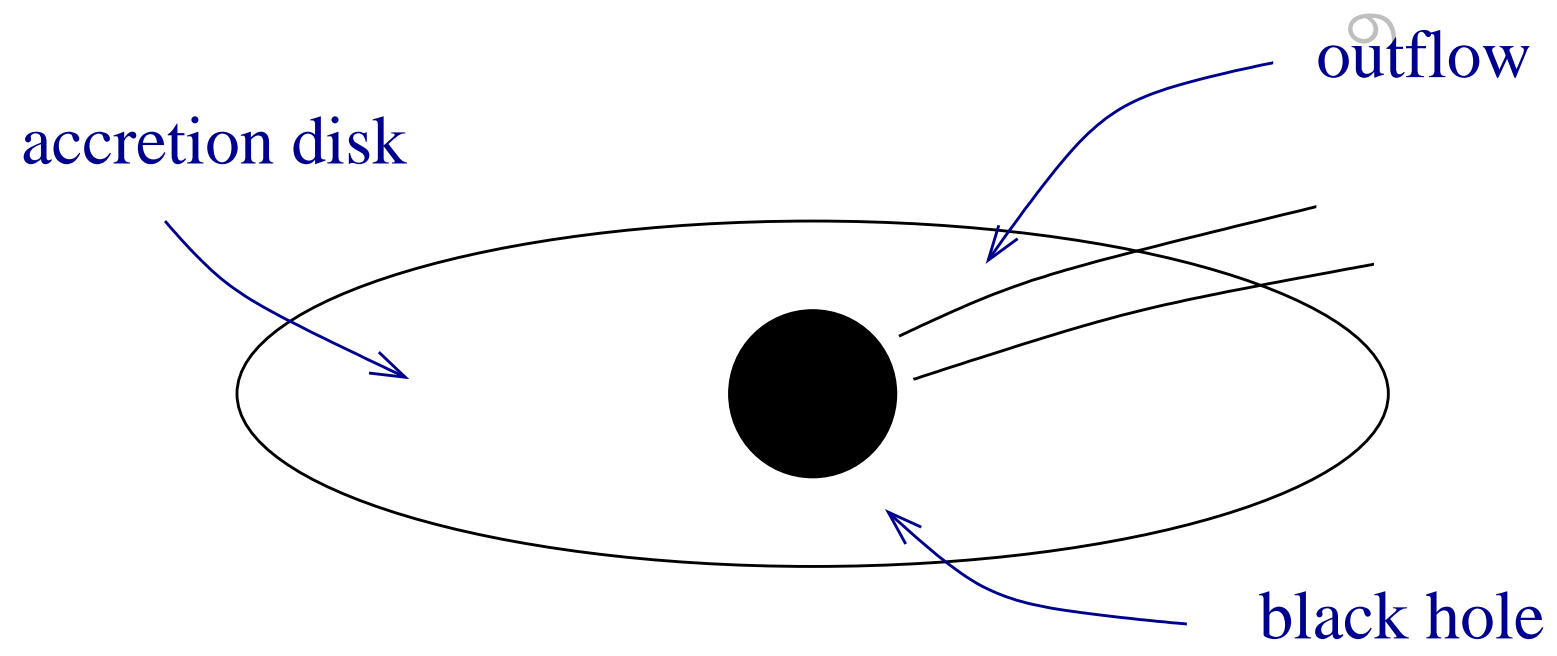


Neutrino Surfaces for the proto-neutron star and the accretion disk

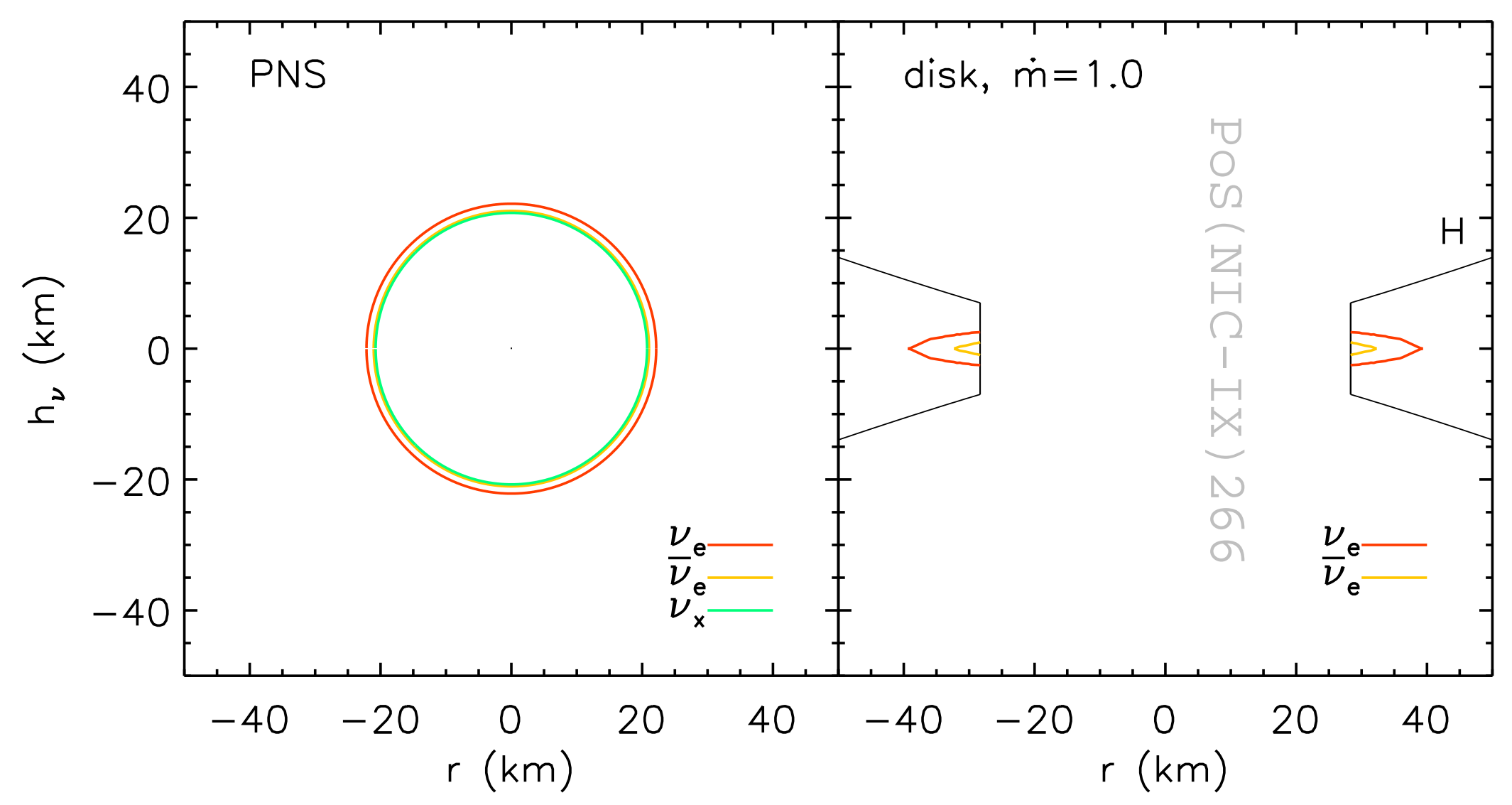

Energies and Spectra of Neutrinos are similar...

Proto-neutron stars have $\nu_{e}, \bar{\nu}_{e}, \nu_{\mu}, \bar{\nu}_{\mu}, \nu_{\tau}, \bar{\nu}_{\tau}$

Disks have primarily $\nu_{e}, \bar{\nu}_{e}$ 


\section{Gamma Ray Bursts}

- characteristics

- few second bursts of $\sim \mathrm{MeV}$ photons, isotropic on the sky

$-10^{51}-10^{54}$ erg if isotropic, but beamed to a few $\%$

- Two classes: Long $(>0.2 \mathrm{~s})$ and short $(<0.2 \mathrm{~s})$ Kouvelietou et al 1993

- Long Bursts - afterglows

- x-ray, radio, optical counterparts, 3 hours-days, months after $\gamma \mathrm{s}$

- SN bumps in a couple of light curves e.g. Stanek eəaे 2003

- Asssociation with Type Ib/c supernovae

- Long Bursts - Association with host galaxies

What astrophysical sites?

Long Bursts - Rare type of Core Collapse SN

Short Bursts - Neutron Star Mergers? 


\section{Accretion Disks around Black Holes may produce Gamma Ray Bursts}

Furthermore, energy deposition from $\nu_{e} \bar{\nu}_{e} \rightarrow e^{+} e^{-}$may be important
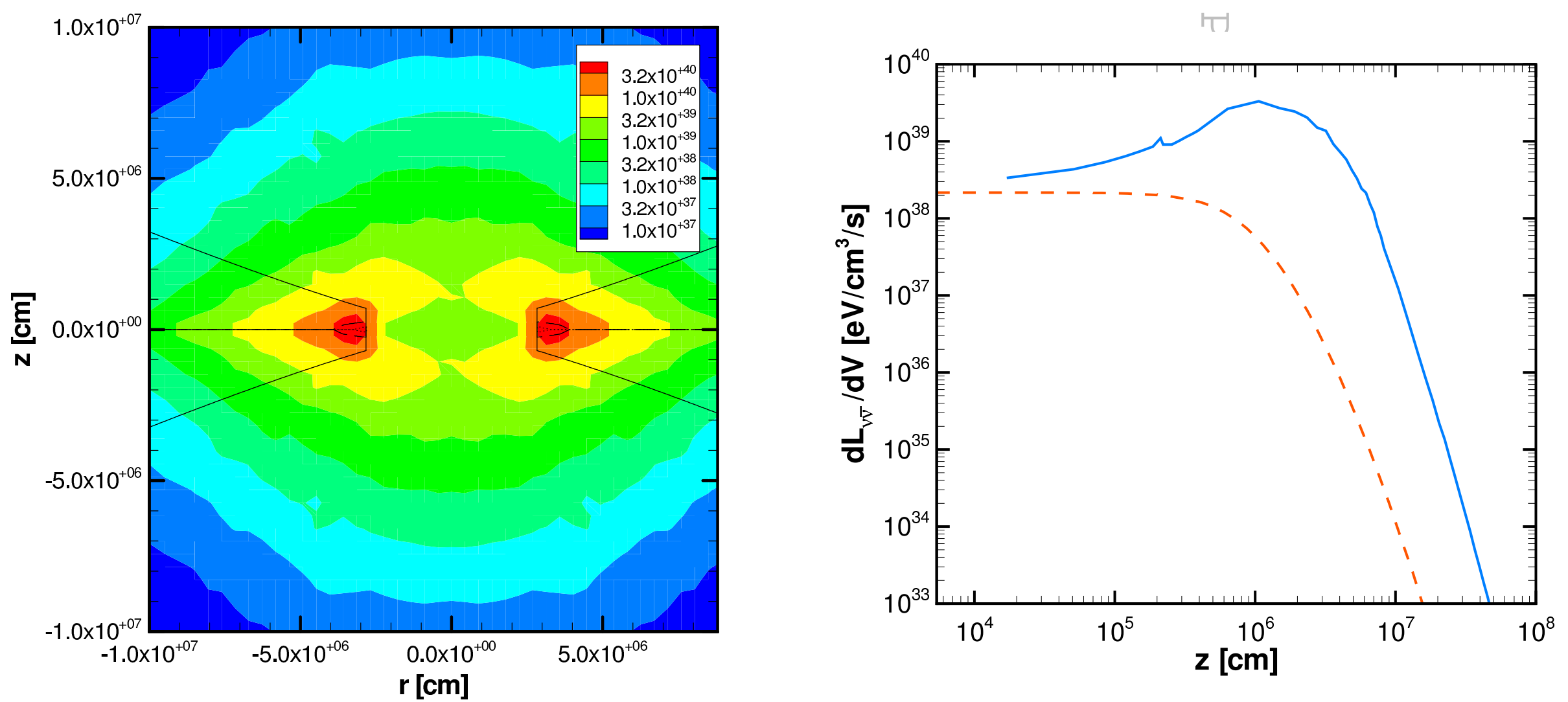

$$
\begin{gathered}
\dot{m}=1 \mathrm{M}_{\odot} \mathrm{s}^{-1}, L_{\nu \bar{\nu}}=10^{50} \operatorname{erg~s}^{-1}, L_{\nu \bar{\nu}} / L_{\nu}=10^{-3} \\
\dot{m}=0.1 \mathrm{M}_{\odot} \mathrm{s}^{-1}, L_{\nu \bar{\nu}}=4 \times 10^{47} \mathrm{erg} \mathrm{s}^{-1}, L_{\nu \bar{\nu}} / L_{\nu}=10^{-4}
\end{gathered}
$$




\section{Galactic Accretion Disk Neutrinos in a Supernova Detector}

How will you know neutrinos from the next Galactic supernova come from a proto-neutron star?

In SuperK:

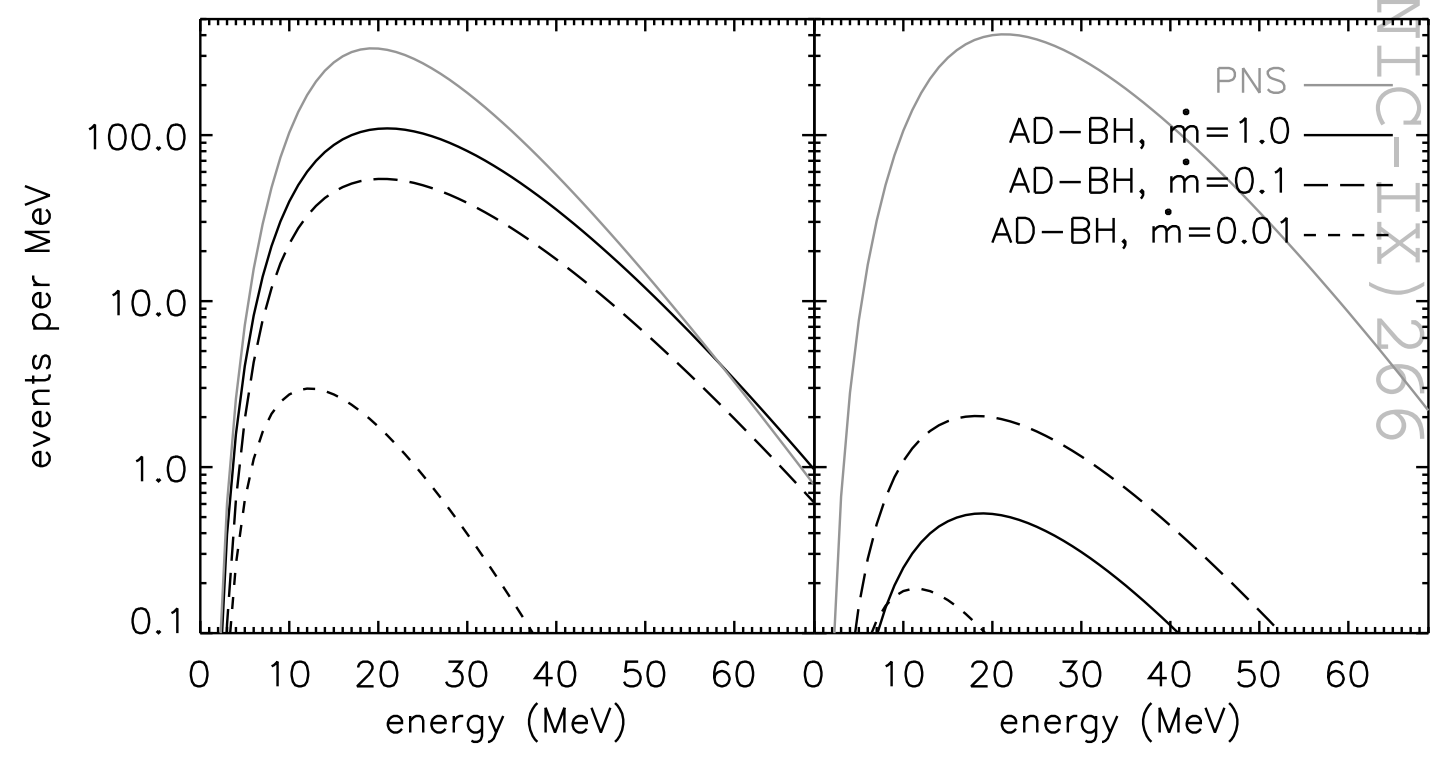

But you can distinguish accretion disks from proto-neutron stars by comparing charged and neutral current signals or looking at time profiles 


\section{Conclusions from this lecture}

- Astrophysical neutrinos can give us information we can't get any other way

- They show up in the early universe, core collapse supernovae, and GRBs

- Future neutrino detections will tell us quite a lot 